\title{
AVALIAÇÃO DO MODELO PRIGOGINE-FLORY- PATTERSON NA PREDIÇÃO DO VOLUME PARCIAL MOLAR DE SOLUÇÕES LÍQUIDAS BINÁRIAS
}

\author{
A.C. GALVÃO, W.S. ROBAZZA, A.M. DA LUZ, L.G. FRANZOSI, R.H. SCHNEIDER \\ Universidade do Estado de Santa Catarina, Departamento de Engenharia de Alimentos \\ E-mail: alessandro.galvao@udesc.br
}

\begin{abstract}
RESUMO - O estudo de grandezas termodinâmicas de soluções fornecem informações importantes sobre as interações moleculares existentes entre os componentes presentes em uma solução. Na realização do presente trabalho foi desenvolvida uma modelagem em software Scilab a partir de dados de volume molar em excesso, disponíveis na literatura, de soluções líquidas binárias formadas por tolueno e n-alcanos a 298,15 K. Os dados de volume molar em excesso foram correlacionados pela equação de Redlich-Kister para geração dos dados de volume parcial molar. As equações do modelo Prigogine-Flory-Patterson foram submetidas a um procedimento aplicando derivadas parciais para fornecer o equacionamento para determinação dos volumes parciais molares. A modelagem foi aplicada para predizer e correlacionar os dados de origem experimental. Foi observado que a aplicação do modelo, com apenas um parâmetro ajustável, fornece bons resultados, tanto na predição como na correlação dos dados de volume parcial molar.
\end{abstract}

\section{INTRODUÇÃO}

O estudo de grandezas termodinâmicas em excesso, como volume molar em excesso $\left(v^{E}\right)$, entalpia molar em excesso $\left(h^{E}\right)$ e energia de Gibbs molar em excesso $\left(g^{E}\right)$ desempenham papel fundamental na compreensão das interações moleculares existentes em soluções. Estas informações também são importantes para o desenvolvimento de teorias de soluções e para o desenvolvimento e teste de modelos matemáticos. Especial atenção é dada ao volume parcial molar $(\bar{v})$, pois essa grandeza defina a real contribuição de cada componente no volume total de uma solução. $\mathrm{O}$ volume parcial molar dos componentes em uma solução $\left(\bar{v}_{i}\right)$ é determinado a partir de dados experimentais de $v^{E}$. Usualmente grandezas em excessos são correlacionadas a modelos matemáticos com a intenção de levantar maiores informações sobre a interação dos componentes em solução. Pouca atenção é dada na determinação e modelagem do $\bar{v}_{i}$, principalmente na avaliação de sua predição utilizando o mesmo conjunto de parâmetros ajustados aos dados de $v^{E}$.

O modelo PFP surgiu da teoria de Prigogine-Flory (Flory, 1965; Orwoll e Flory, 1967 a, b; Flory et al., 1964 a, b; Abe e Flory, 1965; Prigogine et al., 1953 a, b) com adicional refinamento de Patterson e Delmas (1970). O modelo, originalmente desenvolvido para explicar o comportamento de soluções formadas por moléculas apolares, considera a existência de três contribuições: i) contribuição de interação, proporcional ao parâmetro de 
Flory, reflete a variação de energia quando moléculas diferentes estão em contato. ii) contribuição de volume livre, originada na diferença do grau de expansão térmica entre os componentes envolvidos. iii) contribuição de pressão característica, baseada na diferença de pressão interna e volume reduzido dos componentes.

Grandezas termodinâmicas em excesso tem sido correlacionadas com sucesso pelo modelo PFP tanto em soluções envolvendo moléculas apolares como moléculas polares (Checoni e Francesconi, 2006; Galvão e Francesconi, 2008) utilizando um único parâmetro ajustável. Essa característica marcante faz com que mais atenção seja dada ao modelo, todavia não foi encontrado na literatura estudos que associem o modelo ao $\bar{v}_{i}$. Desta forma, este trabalho mostra a capacidade do modelo PFP na predição de dados de $\bar{v}_{i}$ a partir de dados de $v^{E}$.

\section{MÉTODOS}

$\mathrm{Na}$ realização do trabalho a modelagem foi desenvolvida em software Scilab utilizando dados de $v^{E}$ em função da fração molar do tolueno $\left(x_{1}\right)$ de soluções líquidas binárias formadas por tolueno $+n$-alcanos (C8, C9, C10) a 298,15 K publicados por Iloukhani et al., 2006 e por Caceres Alonso et al., 1983. Os dados de $v^{E}$ foram correlacionados pela equação de Redlich-Kister representada pela Equação (1) em que o sistema linear foi resolvido utilizando otimização dos mínimos quadrados.

$$
v^{E}=x_{1}\left(1-x_{1}\right) \sum_{j=0}^{n} A_{j}\left(1-2 x_{1}\right)^{j}
$$

O volume parcial molar dos componentes em uma solução binária é expresso pelas Equações (2) e (3) em que $v$ representa o volume molar.

$$
\begin{aligned}
& \bar{v}_{1}=v^{E}+v_{1}+\left(1-x_{1}\right)\left(\frac{\partial v^{E}}{\partial x_{1}}\right)_{p, T} \\
& \bar{v}_{2}=v^{E}+v_{2}-x_{1}\left(\frac{\partial v^{E}}{\partial x_{1}}\right)_{p, T}
\end{aligned}
$$

A equação de Redlich-Kister quando submetida às Equações (2) e (3) geram as Equações (4) e (5) para o cálculo do $\bar{v}$.

$$
\begin{aligned}
& \bar{v}_{1}=v_{1}+\left(1-x_{1}\right)^{2} \sum_{j=0}^{j=n} A_{j}\left(1-2 x_{1}\right)^{j}-2 x_{1}\left(1-x_{1}\right)^{2} \sum_{j=0}^{j=n} j A_{j}\left(1-2 x_{1}\right)^{j-1} \\
& \bar{v}_{2}=v_{2}+x_{1}^{2} \sum_{j=0}^{j=n} A_{j}\left(1-2 x_{1}\right)^{j}+2 x_{1}^{2}\left(1-x_{1}\right) \sum_{j=0}^{j=n} j A_{j}\left(1-2 x_{1}\right)^{j-1}
\end{aligned}
$$

Os dados de volume parcial molar são determinados aplicando-se os parâmetros gerados pela Equação (1) nas Equações (4) e (5).

O modelo PFP, aplicado na correlação dos dados de $v^{E}$ com um único parâmetro ajustável, é formado por três contribuições conforme representado pela Equação (6). 


$$
\begin{aligned}
& \frac{v^{E}}{G}=I-U+Y \\
& G=\left(x_{1} V_{1}^{*}+x_{2} V_{2}^{*}\right)
\end{aligned}
$$

As contribuições do modelo são conhecidas como contribuição do parâmetro de interação (Equação 8), do volume livre (Equação 9) e da pressão característica (Equação 10).

$$
\begin{aligned}
& I=\frac{\left(\widetilde{V}^{\frac{1}{3}}-1\right) \widetilde{V}^{\frac{2}{3}}}{\left[\left(\frac{4}{3}\right) \widetilde{V}^{-\frac{1}{3}}-1\right]} \theta_{2} \psi_{1}\left[\frac{\chi_{12}}{P_{1}^{*}}\right] \\
& U=\frac{\left(\widetilde{V}_{1}-\widetilde{V}_{2}\right)^{2}\left[\left(\frac{14}{9}\right) \widetilde{V}^{-\frac{1}{3}}-1\right]}{\left[\left(\frac{4}{3}\right) \widetilde{V}^{-\frac{1}{3}}-1\right] \widetilde{V}} \psi_{1} \psi_{2} \\
& Y=\frac{\left(\widetilde{V}_{1}-\widetilde{V}_{2}\right)\left(P_{1}^{*}-P_{2}^{*}\right)}{P_{1}^{*} \psi_{2}+P_{2}^{*} \psi_{1}} \psi_{1} \psi_{2}
\end{aligned}
$$

Para o uso do modelo PFP é necessário o conhecimento de informações dos componentes puros; coeficiente de expansão térmica $\left(\alpha_{i}\right)$, coeficiente de compressibilidade isotérmica $\left(\beta_{i}\right)$ e volume molar $\left(v_{i}\right)$. Os dados dos componentes puros utilizados neste trabalho são apresentados na Tabela 1 . Os volumes reduzidos $\left(\tilde{V}_{i}\right)$ para cada componente são calculados pela Equação (11).

$$
\tilde{V}_{i}=\left(\frac{1+\left(\frac{4}{3}\right) \alpha_{i} T}{1+\alpha_{i} T}\right)^{3}
$$

Tabela 1 - Dados dos componentes puros; coeficientes de expansão térmica $(\alpha)$, coeficiente de compressibilidade isotérmica $(\beta)$ e volume molar $(v)$

\begin{tabular}{cccc}
\hline & $\mathbf{1 0}^{\mathbf{3}} \cdot \boldsymbol{\beta}\left(\mathbf{M P a} \mathbf{a}^{\mathbf{1}}\right)$ & $\mathbf{1 0}^{\mathbf{3}} \cdot \boldsymbol{\alpha}\left(\mathbf{K}^{\mathbf{- 1}}\right)$ & $\boldsymbol{v}\left(\mathbf{c m}^{\mathbf{3}} \cdot \mathbf{m o l}^{\mathbf{- 1}}\right)$ \\
\hline Tolueno & 0,9110 & 1,0710 & 106,9145 \\
Octano & 1,3024 & 1,1640 & 163,5100 \\
Nonano & 1,1754 & 1,0900 & 178,7900 \\
Decano & 1,1096 & 1,0500 & 195,9200 \\
\hline
\end{tabular}

A partir dos resultados encontrados para volumes reduzidos, juntamente com o coeficiente de compressibilidade do componente puro e o volume molar, é calculada a pressão característica de cada componente $\left(P_{i}^{*}\right)$.

$$
P_{i}^{*}=\frac{\alpha_{i} T v_{i}^{2}}{\beta_{i}}
$$

O cálculo do volume característico para cada componente $\left(V_{i}^{*}\right)$ é determinado conhecendo-se o $v_{i}$ e o $\tilde{V}_{i}$ conforme apresentado na Equação (13). 


$$
V_{i}^{*}=\frac{V_{i}}{\widetilde{V}_{i}}
$$

As frações volumétricas dos componentes em solução $\left(\emptyset_{1}\right)$ são calculadas a partir do $V_{i}^{*}$ e das frações molares dos componentes na solução $\left(x_{1}, x_{2}\right)$, apresentado na Equação (14).

$$
\emptyset_{1}=1-\emptyset_{2}=\frac{x_{1} V_{1}^{*}}{x_{1} V_{1}^{*}+x_{2} V_{2}^{*}}
$$

As frações volumétricas obtidas são utilizadas no cálculo das frações de energia de contato dos componentes em solução $\left(\psi_{i}\right)$.

$$
\psi_{1}=1-\psi_{2}=\frac{\emptyset_{1} P_{1}^{*}}{\emptyset_{1} P_{1}^{*}+\emptyset_{2} P_{2}^{*}}
$$

O volume reduzido da solução $(\tilde{V})$ é calculado pela Equação (16). A fração de superfície dos componentes $\left(\theta_{i}\right)$ é calculada pela Equação (17) em que $s_{1}$ e $s_{2}$ são a área superficial das moléculas envolvidas e sua relação é calculada pela Equação (18).

$$
\begin{aligned}
& \tilde{V}=\psi_{1} \tilde{V}_{1}+\psi_{2} \tilde{V}_{2} \\
& \theta_{1}=1-\theta_{2}=\frac{\emptyset_{1}}{\left[\emptyset_{1}+\left(\frac{s_{2} \phi_{2}}{s_{1}}\right)\right]} \\
& \frac{s_{1}}{s_{2}}=\left(\frac{V_{1}^{*}}{V_{2}^{*}}\right)^{-\frac{1}{3}}
\end{aligned}
$$

Para a determinação dos volumes parciais molares utilizando o parâmetro ajustável do modelo $\left(\chi_{12}\right)$, determinado a partir da correlação do modelo aos dados de $v^{E}$, as equações do PFP foram submetidas as Equações (2) e (3) necessitando a derivada parcial de $v^{E}$ em relação a $x_{1}$ como expresso pelas Equações (19) a (22).

$$
\begin{aligned}
& \frac{\partial v^{E}}{\partial x_{1}}=\frac{\partial I}{\partial x_{1}}-\frac{\partial U}{\partial x_{1}}+\frac{\partial Y}{\partial x_{1}} \\
& \frac{\partial I}{\partial x_{1}}=\frac{\partial I}{\partial \widetilde{V}} \cdot \frac{d \widetilde{V}}{d \psi_{1}} \cdot \frac{d \psi_{1}}{d \phi_{1}} \cdot \frac{d \phi_{1}}{d x_{1}}+\frac{\partial I}{\partial \psi_{1}} \cdot \frac{d \psi_{1}}{d \phi_{1}} \cdot \frac{d \phi_{1}}{d x_{1}}+\frac{\partial I}{\partial \theta_{2}} \cdot \frac{d \theta_{2}}{d x_{1}}+\frac{\partial I}{\partial G} \cdot \frac{d G}{d x_{1}} \\
& \frac{\partial U}{\partial x_{1}}=\frac{\partial U}{\partial \widetilde{V}} \cdot \frac{d \widetilde{V}}{d \psi_{1}} \cdot \frac{d \psi_{1}}{d \phi_{1}} \cdot \frac{d \phi_{1}}{d x_{1}}+\frac{\partial U}{\partial \psi_{1}} \cdot \frac{d \psi_{1}}{d \phi_{1}} \cdot \frac{d \phi_{1}}{d x_{1}}+\frac{\partial U}{\partial G} \cdot \frac{d G}{d x_{1}} \\
& \frac{\partial Y}{\partial x_{1}}=\frac{\partial Y}{\partial \psi_{1}} \cdot \frac{d \psi_{1}}{d \phi_{1}} \cdot \frac{d \phi_{1}}{d x_{1}}+\frac{\partial Y}{\partial G} \cdot \frac{d G}{d x_{1}}
\end{aligned}
$$

Além da utilização do modelo PFP na predição dos resultados de $\bar{v}_{i}$, o equacionamento também foi aplicado na correlação dos dados de $\bar{v}_{i}$, de forma que a determinação do parâmetro ajustável foi feita a partir da minimização da função objetivo expressa pela Equação (23) na qual $N_{p}$ representa o número de pontos experimentais. 


$$
\text { F.O. }=\sum_{i=1}^{N_{p}}\left(\frac{\bar{v}_{1(\exp )}-\bar{v}_{1(P F P)}}{\bar{v}_{1(\exp )}}\right)_{i}^{2}+\sum_{i=1}^{N_{p}}\left(\frac{\bar{v}_{2(\exp )}-\bar{v}_{2(P F P)}}{\bar{v}_{2(\exp )}}\right)_{i}^{2}
$$

Para realizar a comparação entre os resultados obtidos de forma preditiva e os resultados obtidos de forma correlativa, em relação aos dados experimentais, foram utilizados cálculos de desvio relativo médio (ARD) representado pela Equação (24).

$$
A R D=\frac{100}{N p} \sum_{i=1}^{N p}\left|\frac{\bar{v}_{(\exp )_{i}}-\bar{v}_{(P F P)_{i}}}{\bar{v}_{(\exp )_{i}}}\right|
$$

\section{RESULTADOS E DISCUSSÕES}

Os parâmetros da equação de Redlich-Kister determinados na correlação dos dados de volume molar em excesso para todas as soluções binárias estudadas são apresentados na Tabela 2.

Tabela 2 - Coeficientes gerados pela equação de Redlich-Kister e os respectivos desvios

\begin{tabular}{|c|c|c|c|c|c|}
\hline $\begin{array}{c}\mathbf{A}_{0} \\
\left(\mathrm{~cm}^{3 \cdot} \mathrm{mol}^{-1}\right)\end{array}$ & $\begin{array}{c}\mathbf{A}_{1} \\
\left(\mathrm{~cm}^{3 \cdot} \mathrm{mol}^{-1}\right)\end{array}$ & $\begin{array}{c}\begin{array}{c}\mathbf{A}_{2} \\
\left(\mathrm{~cm}^{3 \cdot} \mathrm{mol}^{-1}\right)\end{array} \\
\end{array}$ & $\begin{array}{c}\mathbf{A}_{3} \\
\left(\mathbf{c m}^{3} \cdot \mathbf{m o l}^{-1}\right) \\
\end{array}$ & $\begin{array}{c}\mathbf{A}_{4} \\
\left(\mathrm{~cm}^{3 \cdot} \mathrm{mol}^{-1}\right)\end{array}$ & $\begin{array}{c}\sigma \\
\left(\mathrm{cm}^{3} \mathbf{m o l}^{-1}\right) \\
\end{array}$ \\
\hline \multicolumn{6}{|c|}{ Tolueno + Octano } \\
\hline 0,9854 & $-0,1207$ & 0,1711 & 0,0606 & $-0,1154$ & 0,0015 \\
\hline 1,3745 & $-0,1732$ & $\begin{array}{l}\text { Toluen } \\
-0,1802\end{array}$ & $\begin{array}{l}\text { Jonano } \\
-0,0013\end{array}$ & 0,0214 & 0,0004 \\
\hline 1,5653 & $-0,4279$ & Tolueno + Decano & $\begin{array}{l}\text { Pecano } \\
-0,0413\end{array}$ & 0,0098 & 0,0013 \\
\hline
\end{tabular}
padrões $(\sigma)$ em relação aos dados de volume molar em excesso

Na Tabela 3 são apresentados os valores do parâmetro ajustável do modelo PFP obtidos na correlação dos dados de volume molar em excesso e utilizados na predição dos valores de volume parcial molar bem como valores do parâmetro ajustável obtidos na correlação dos dados de volume parcial molar juntamente com o desvio existente entre os dados experimentais e os dados da modelagem.

Tabela 3 - Parâmetros do modelo PFP $\left(\chi_{12}\right)$ e desvios relativos médios $(A R D)$ para as soluções formadas por tolueno $+n$-alcano na modelagem preditiva (p) e correlativa (c)

\begin{tabular}{ccccccc}
\hline & $\chi_{\mathbf{1 2}(\mathbf{p})}\left(\mathbf{J} . c m^{-\mathbf{3}}\right)$ & $\chi_{\mathbf{1 2}(\mathbf{c})}\left({\left.\mathbf{J} . \mathbf{c m}^{-3}\right)}^{-3}\right.$ & $\boldsymbol{A R D 1}_{(\mathbf{p})}$ & $\boldsymbol{A R D 2}_{(\mathbf{p})}$ & $\boldsymbol{A R D 1}_{(\mathbf{c})}$ & $\boldsymbol{A R D 2}_{(\mathbf{c})}$ \\
\hline Octano & 18,30 & 17,54 & 0,0177 & 0,0248 & 0,0063 & 0,0210 \\
Nonano & 17,48 & 15,25 & 0,0732 & 0,0136 & 0,0254 & 0,0397 \\
Decano & 18,16 & 14,06 & 0,1241 & 0,0303 & 0,0502 & 0,0275 \\
\hline
\end{tabular}

Os resultados obtidos da aplicação do modelo PFP na predição e na correlação dos dados de volume parcial molar em comparação com os valores de origem experimental são apresentados na Tabela 4. 
Tabela 4 - Comparação dos resultados de volume parcial molar em relação aos resultados preditivos (p) e correlativos (c) para todos as soluções binárias estudadas

\begin{tabular}{|c|c|c|c|c|c|c|}
\hline$x_{1}$ & $\begin{array}{c}\bar{v}_{1} \\
\left(\mathrm{~cm}^{3} \cdot \mathrm{mol}^{-1}\right)\end{array}$ & $\begin{array}{c}\bar{v}_{1} \operatorname{PFP}(\mathbf{p}) \\
\left(\mathrm{cm}^{3} \cdot \mathrm{mol}^{-1}\right)\end{array}$ & $\begin{array}{c}\bar{v}_{1} \mathrm{PFP}(\mathrm{c}) \\
\left(\mathrm{cm}^{3} \cdot \mathrm{mol}^{-1}\right)\end{array}$ & $\begin{array}{c}\bar{v}_{2} \\
\left(\mathbf{c m}^{3} \cdot \mathbf{m o l}^{-1}\right) \\
\end{array}$ & $\begin{array}{c}\bar{v}_{2 \operatorname{PFP}(\mathbf{p})} \\
\left(\mathrm{cm}^{3} \cdot \mathrm{mol}^{-1}\right)\end{array}$ & $\begin{array}{c}\bar{v}_{2 \mathrm{PFP}(\mathrm{c})} \\
\left(\mathrm{cm}^{3} \cdot \mathrm{mol}^{-1}\right)\end{array}$ \\
\hline \multicolumn{7}{|c|}{ Tolueno (1) + Octano (2) } \\
\hline 0,0461 & 107,8092 & 107,8988 & 107,8394 & 163,5121 & 163,5125 & 163,5124 \\
\hline 0,1173 & 107,6861 & 107,7441 & 107,6935 & 163,5245 & 163,5263 & 163,5254 \\
\hline 0,2428 & 107,4812 & 107,5051 & 107,4682 & 163,5707 & 163,5787 & 163,5747 \\
\hline 0,3395 & 107,3333 & 107,3502 & 107,3225 & 163,6201 & 163,6422 & 163,6345 \\
\hline 0,3478 & 107,3213 & 107,3381 & 107,3111 & 163,6248 & 163,6486 & 163,6405 \\
\hline 0,4402 & 107,1981 & 107,2158 & 107,1962 & 163,6817 & 163,7280 & 163,7151 \\
\hline 0,5099 & 107,1206 & 107,1385 & 107,1236 & 163,7344 & 163,7979 & 163,7807 \\
\hline 0,5584 & 107,0756 & 107,0921 & 107,0801 & 163,7790 & 163,8511 & 163,8306 \\
\hline 0,6054 & 107,0389 & 107,0528 & 107,0433 & 163,8308 & 163,9057 & 163,8817 \\
\hline 0,6260 & 107,0250 & 107,0373 & 107,0288 & 163,8567 & 163,9306 & 163,9050 \\
\hline 0,6590 & 107,0051 & 107,0146 & 107,0076 & 163,9026 & 163,9714 & 163,9431 \\
\hline 0,7118 & 106,9791 & 106,9836 & 106,9787 & 163,9887 & 164,0388 & 164,0061 \\
\hline 0,7567 & 106,9616 & 106,9622 & 106,9587 & 164,0744 & 164,0979 & 164,0611 \\
\hline 0,7656 & 106,9585 & 106,9585 & 106,9553 & 164,0927 & 164,1098 & 164,0722 \\
\hline 0,8141 & 106,9440 & 106,9411 & 106,9391 & 164,1994 & 164,1749 & 164,1328 \\
\hline 0,8673 & 106,9313 & 106,9274 & 106,9265 & 164,3258 & 164,2470 & 164,1996 \\
\hline \multicolumn{7}{|c|}{ Tolueno $(1)+$ Nonano $(2)$} \\
\hline 0,0396 & 107,8752 & 108,1138 & 107,9505 & 178,7904 & 178,7918 & 178,7916 \\
\hline 0,1938 & 107,6021 & 107,7866 & 107,6675 & 178,8125 & 178,8354 & 178,8292 \\
\hline 0,2815 & 107,4733 & 107,6185 & 107,5222 & 178,8533 & 178,8878 & 178,8746 \\
\hline 0,3621 & 107,3689 & 107,4770 & 107,3999 & 178,9168 & 178,9550 & 178,9327 \\
\hline 0,4429 & 107,2753 & 107,3487 & 107,2891 & 179,0084 & 179,0415 & 179,0074 \\
\hline 0,6000 & 107,1192 & 107,1424 & 107,1109 & 179,2653 & 179,2668 & 179,2019 \\
\hline 0,6627 & 107,0662 & 107,0773 & 107,0548 & 179,3926 & 179,3784 & 179,2981 \\
\hline 0,7595 & 106,9966 & 106,9975 & 106,9860 & 179,6079 & 179,5748 & 179,4675 \\
\hline 0,8399 & 106,9526 & 106,9512 & 106,9461 & 179,7972 & 179,7596 & 179,6267 \\
\hline 0,9168 & 106,9252 & 106,9244 & 106,9230 & 179,9812 & 179,9536 & 179,7936 \\
\hline \multicolumn{7}{|c|}{ Tolueno (1) + Decano (2) } \\
\hline 0,0924 & 107,9484 & 108,1532 & 107,8907 & 195,9274 & 195,9301 & 195,9279 \\
\hline 0,2486 & 107,6114 & 107,8239 & 107,6317 & 195,9776 & 195,9984 & 195,9817 \\
\hline 0,3462 & 107,4310 & 107,6330 & 107,4814 & 196,0394 & 196,0795 & 196,0455 \\
\hline 0,3707 & 107,3896 & 107,5872 & 107,4454 & 196,0595 & 196,1050 & 196,0656 \\
\hline 0,4692 & 107,2400 & 107,4137 & 107,3087 & 196,1632 & 196,2310 & 196,1648 \\
\hline 0,5184 & 107,1756 & 107,3340 & 107,2459 & 196,2312 & 196,3087 & 196,2261 \\
\hline 0,5465 & 107,1421 & 107,2909 & 107,2118 & 196,2759 & 196,3579 & 196,2649 \\
\hline 0,5875 & 107,0974 & 107,2311 & 107,1647 & 196,3497 & 196,4361 & 196,3266 \\
\hline 0,6024 & 107,0824 & 107,2104 & 107,1484 & 196,3792 & 196,4665 & 196,3506 \\
\hline 0,6393 & 107,0483 & 107,1617 & 107,1099 & 196,4594 & 196,5464 & 196,4136 \\
\hline 0,6536 & 107,0362 & 107,1437 & 107,0958 & 196,4934 & 196,5792 & 196,4395 \\
\hline 0,6960 & 107,0039 & 107,0939 & 107,0565 & 196,6047 & 196,6826 & 196,5211 \\
\hline 0,7182 & 106,9892 & 107,0700 & 107,0375 & 196,6698 & 196,7404 & 196,5668 \\
\hline 0,7337 & 106,9798 & 107,0541 & 107,0250 & 196,7183 & 196,7824 & 196,5999 \\
\hline 0,7645 & 106,9632 & 107,0249 & 107,0019 & 196,8228 & 196,8696 & 196,6689 \\
\hline 0,8105 & 106,9435 & 106,9872 & 106,9720 & 197,0010 & 197,0097 & 196,7797 \\
\hline
\end{tabular}


Com a finalidade de ilustrar o comportamento da modelagem, a Figura 1 compara resultados experimentais e resultados do modelo para algumas soluções analisadas.

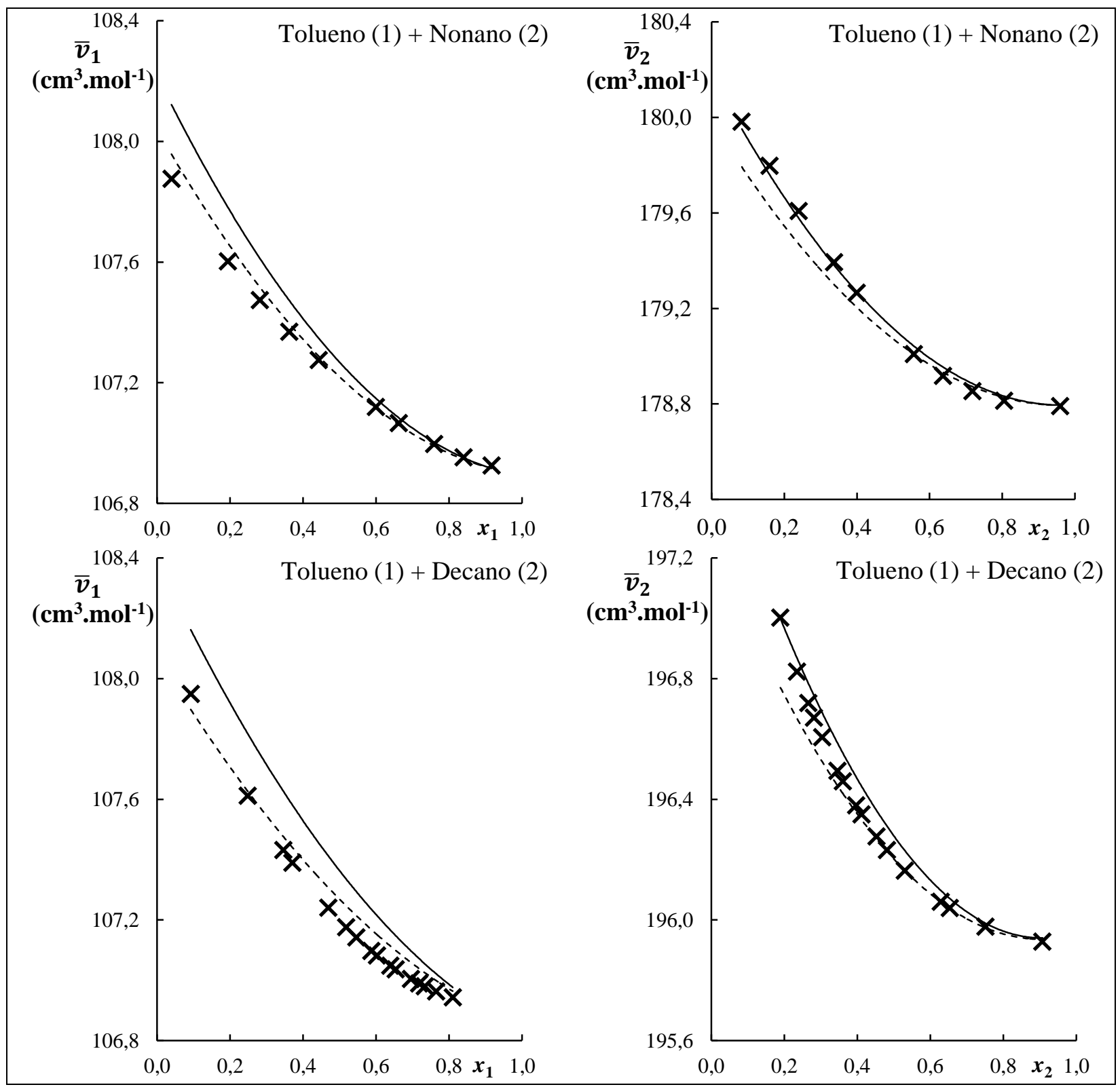

Figura - Comportamento do volume parcial molar em função da fração molar da solução: $\times$ dados experimentais; - PFP preditivo; - - - PFP correlativo

Avaliando os resultados de uma forma geral, observa-se que o modelo PFP apresentou ótimo desempenho na determinação de dados de volume parcial molar para as soluções estudadas, tanto aplicado de forma preditiva como de forma correlativa.

Uma análise dos valores de desvio relativo médio indicam que para a pior condição observada o desvio foi da ordem de $0,12 \%$ e a melhor condição observada apresentou um desvio de $0,006 \%$. Observa-se que para todas as soluções estudadas o volume parcial molar do tolueno foi melhor representado utilizando o modelo PFP na forma correlativa. Para os $n$ - 
alcanos, os desvios existentes na correlação e na predição dos dados são bastante próximos porém, para o nonano melhores resultados são obtidos na abordagem preditiva e para o octano e decano na forma correlativa.

\section{CONCLUSÕES}

O modelo Prigogine-Flory-Patterson apresenta habilidade na determinação de dados de volume parcial molar de soluções líquidas binárias formadas por moléculas apolares. Os resultados obtidos, tanto com abordagem preditiva como com abordagem correlativa, são bastante próximos evidenciando a capacidade do modelo devido a seu embasamento teórico bem estabelecido. Futuros estudos devem ser conduzidos, todavia existe indicio que o modelo PFP possa ser aplicado em situações práticas ou no desenvolvimento de novos equacionamentos em que o volume da solução líquida deva ser determinado com maior precisão.

\section{REFERÊNCIAS}

ABE, A.; FLORY, P.J. The thermodynamic properties of mixtures of small, nonpolar molecules. J. Am. Chem. Soc., v. 87, p.1838-1846, 1965.

CACERES ALONSO, M.; POVEDA VILCHES, J.L.; SANCHEZ-PAJARES, R.G.; NUNEZ DELGADO, J. Excess volumes of (toluene + n-alkane)s. J. Chem. Thermodynamics v. 15, p.913-917, 1983.

CHECONI, R.F.; FRANCESCONI, A.Z. Partial molar enthalpy properties and correlation of excess molar enthalpy data of acetonitrile + diethylamine or S-butylamine mixtures at various temperatures and atmospheric pressure. Thermochim. Acta, v. 450, p.126-131, 2006.

FLORY, P.J.; ORWOLL, R.A.; VRIJ, A. Statistical thermodynamics of chain molecule liquids. I An equation of state for normal paraffin hydrocarbons. J. Am. Chem. Soc., v. 86, p.3507-3514, 1964 a.

FLORY, P.J.; ORWOLL, R.A.; VRIJ, A. Statistical thermodynamics of chain molecule liquids. II Liquid mixtures of normal paraffin hydrocarbons. J. Am. Chem. Soc., v. 86, p.3515-3520, 1964 b.

FLORY, P.J. Statistical thermodynamics of liquid mixtures. J. Am. Chem. Soc., v. 87, p.1833$1838,1965$.

GALVÃO, A.C.; FRANCESCONI, A.Z. Application of the Prigogine-Flory-Patterson model to excess molar enthalpy of binary liquid mixtures containing acetonitrile and 1-alkanol. J. Mol. Liq., v. 139, p.110-116, 2008. 
ILOUKHANI, H.; REZAEI-SAMETI, M.; BASIRI-PARSA, J. Excess molar volumes and dynamic viscosities for binary mixtures of toluene $+n$-alkanes $(C 5-C 10)$ at $\mathrm{T}=298.15$ $\mathrm{K}$ - Comparison with Prigogine-Flory-Patterson theory. J. Chem. Thermodynamics v. 38, p.975-982, 2006.

ORWOLL, R.A.; FLORY, P.J. Thermodynamics properties of binary mixtures of n-alkanes. J. Am. Chem. Soc., v. 89, p.6822-6829, 1967 a.

ORWOLL, R.A.; FLORY, P.J. Equation of state parameter for normal alkanes. Correlation with chain length. J. Am. Chem. Soc., v. 89, p.6814-6822, 1967 b.

PATTERSON, D.; DELMAS, G. Corresponding states theories and liquid models. Disc. Faraday Soc., v. 49, p.98-105, 1970.

PRIGOGINE, I.; TRAPPENIERS, N.; MATHOT, V. Statistical thermodynamics of r-mers and r-mer solutions. Dis. Faraday Soc., v. 15, p.93-107, 1953 a.

PRIGOGINE, I.; TRAPPENIERS, N.; MATHOT, V. J. On the application of the cell method to r-mer liquids. Chem. Phys., v. 21, p.559-560, 1953 b. 